\title{
Optimization of LMP-specific CTL expansion for potential adoptive immunotherapy in NPC patients
}

\author{
Viviana P Lutzky ${ }^{1,6}$, Joanne E Davis ${ }^{1,2,6}$, Pauline Crooks ${ }^{1}$, Monika Corban ${ }^{1}$, Mark C Smith ${ }^{1}$, Michael Elliott ${ }^{1}$, \\ Leanne Morrison $^{1}$, Simone Cross ${ }^{1}$, David Tscharke ${ }^{3}$, Benedict Panizza ${ }^{4}$, William Coman ${ }^{4}$, Mandvi Bharadwaj ${ }^{5}$ \\ and Denis J Moss ${ }^{1}$
}

\begin{abstract}
Nasopharyngeal carcinoma (NPC) is Epstein-Barr virus (EBV) positive in all undifferentiated cases, expressing the latency II phenotype of latent membrane proteins (LMPs) 1 and 2, in addition to EBV nuclear antigen (EBNA) 1. Several studies have attempted to treat NPC with EBV-specific cytotoxic T lymphocyte (CTL) with a partial response. To improve this therapy, there is a need to expand CTL targeted to the latency II antigens of EBV, rather than the immunodominant EBV nuclear antigens 3-6 peptides typically expanded by lymphoblastoid cells. In order to maximize the expansion of LMP-specific CTL in vitro for use in adoptive immunotherapy of nasopharyngeal carcinoma patients, we used lymphoblastoid cell lines coated with synthetic peptides corresponding to CTL determinants from the LMP proteins. We investigated several issues pertaining to the expansion of an immunologically weak CTL response, including peptide and interleukin-2 concentration, and screening assays for selecting the optimal peptide for use in expansion of LMP-specific CTL. Although screening of ex vivo peripheral blood mononuclear cells did not prove to be useful in the selection of an LMP peptide for use in CTL cultures, the peptide and interleukin-2 concentrations were critical for the maximum expansion of CTL. Therefore, it is imperative that stimulation protocols are optimized for the expansion of LMP-specific CTL.

Immunology and Cell Biology (2009) 87, 481-488; doi:10.1038/icb.2009.25; published online 26 May 2009
\end{abstract}

Keywords: Epstein-Barr virus; interleukin-2; latent membrane protein-specific CTL; optimization of cell expansion; peptide avidity

Epstein-Barr virus (EBV) is a $\gamma$-herpesvirus, which infects up to $90 \%$ of the adult population, and persists for life as a latent infection in resting B cells. ${ }^{1}$ After primary infection, EBV is controlled by a potent cytotoxic T-lymphocyte (CTL) response, primarily to the lytic and a subset of EBV latent nuclear antigens (EBNAs) 3-6. The virus is also associated with a number of malignancies, including post-transplant lymphoproliferative disease (PTLD), Hodgkin's lymphoma (HL), Burkitt's lymphoma (BL) and nasopharyngeal carcinoma (NPC). These tumours can arise because of immunosuppression (PTLD), or through evasion of the immune response, particularly by downregulation of EBV protein expression on the cell surface (NPC, HL and BL). EBV-induced malignancies have been classified into three categories, termed latency I, II and III, according to EBV protein expression. Thus, PTLD is a latency III tumour, as it expresses EBNAs 1-6, and the latent membrane proteins (LMPs) 1 and 2. Latency III tumours are most likely to respond to EBV-specific CTL therapies, as the EBNAs
3-6 proteins are particularly immunodominant. NPC and HL are latency II tumours, and express EBNA 1 and LMPs 1 and 2 only, which are relatively weak at inducing CTL responses. ${ }^{1}$

This report focuses on the problem of developing novel immunotherapies for NPC, which is relatively common in southern China, South-East Asia, North Africa and northern Europe. This malignancy is a good candidate for immunotherapy as all undifferentiated NPC tumours are EBV positive. ${ }^{1}$ Adoptive immunotherapy for the treatment of PTLD in bone marrow and solid organ transplant patients has been successfully used for over 10 years, using autologous EBV-immortalized lymphoblastoid cell lines (LCLs) to stimulate the expansion of EBV-specific CTL. ${ }^{2,3}$ LCLs are the perfect antigenpresenting cell in this model, as they express the immunodominant EBV proteins, EBNAs 3-6, and have a latency III phenotype. ${ }^{1}$ Few side effects of adoptive immunotherapy using autologous EBV-specific CTL have been reported and multiple infusions are well tolerated,

\footnotetext{
${ }^{1}$ EBV Biology Laboratory, Division of immunology, Australian Centre for Vaccine Development, The Queensland Institute of Medical Research, The Royal Brisbane Hospital, Herston, Brisbane, Queensland, Australia; ${ }^{2}$ The Cancer Immunology Research Program, The Peter MacCallum Cancer Institute, East Melbourne, Melbourne, Victoria, Australia; ${ }^{3}$ School of Biochemistry and Molecular Biology, Australia National University, Canberra, Australia; ${ }^{4}$ Head and Neck Clinic, The Princess Alexandra Hospital, Woolloongabba, Brisbane, Queensland, Australia and ${ }^{5}$ mmunology and Microbiology Department, University of Melbourne, Parkville, Melbourne, Victoria, Australia

${ }^{6}$ These authors contribute equally to this work.

Correspondence: DrVP Lutzky, Queensland Institute of Medical Research, Australian Centre for Vaccine Development, The Royal Brisbane Hospital, 300 Herston Road, Brisbane, 4006, Australia.

E-mail: viviana.lutzky@qimr.edu.au

Received 26 November 2008; revised 20 February 2009; accepted 25 March 2009; published online 26 May 2009
} 
inducing tumour regression and long-term protection against recurrent disease. Adoptive transfer of EBV-specific CTL also contributes to reduction of EBV DNA levels, and an increase of EBV-specific CTL in the peripheral blood. ${ }^{4}$ On the basis of the success of treating PTLD, several studies have investigated the potential of autologous LCLexpanded CTL to regress advanced NPC or relapsed HL tumours. In these cases, the dominant CTL responses were directed towards EBNAs 3-6 proteins, which are not expressed on NPC tumours, although variable LMP 2 responses have been raised in the majority of patient cell lines using this method. ${ }^{5}$ EBV levels decreased and there was some resolution of symptoms in patients treated with LCLstimulated CTL. ${ }^{6-8}$ Meanwhile, other studies have used an alternative approach based on the in vitro stimulation of CTLs with dendritic cells. ${ }^{9-12}$

The present study arose out of our aim to conduct a clinical trial on NPC patients with effector cells specifically activated against determinants expressed in NPC cells. Preliminary results (unreported) suggested that peptide-coated LCLs, rather than other methods of stimulation, activated the strongest LMPs 1 and 2 responses. This report summarizes our experience in investigating the optimal conditions for activating LMPs 1 and 2 responses using defined peptide epitopes in cultures from healthy individuals and NPC patients (Table 1).

Table 1 EBV peptides used in this study

\begin{tabular}{lllll}
\hline EBV protein & $\begin{array}{l}\text { Peptide } \\
\text { sequence }\end{array}$ & Abbreviation & $\begin{array}{l}\text { HLA } \\
\text { restriction }\end{array}$ & Reference \\
\hline LMP1 & ALLVLYSFA & ALL & A2 & Duraiswamy J et al. ${ }^{13}$ \\
LMP2 & CLGGLLTMV & CLG & A2 & Lee SP et al. ${ }^{14}$ \\
LMP2 & FLYALALL & FLY & A2 & Lautscham G et al. ${ }^{15}$ \\
LMP1 & IALYLQQNW & IAL & B57, B58 & Duraiswamy J et al. ${ }^{13}$ \\
LMP2 & IEDPPFNSL & IED & B40, B60 & Lee SP et al. ${ }^{16}$ \\
LMP2 & LLSAWILTA & LLS & A2 & Lee SP et al. ${ }^{17}$ \\
LMP2 & LTAGFLIFL & LTA & A2 & Lee SP et al. ${ }^{16}$ \\
LMP2 & PYLFWLAAI & PYL & A23 & Khanna R et al. ${ }^{18}$ \\
LMP2 & SSCSSCPLSK & SSC & A11 & Lee SP et al. ${ }^{16}$ \\
LMP2 & TYGPVFMCL & TYG & A24 & Lee SP et al. ${ }^{16}$ \\
LMP1 & YLLEMLWRL & YLL & A2, A68, A69 & Khanna R et al. ${ }^{19}$ \\
LMP1 & YLQQNWWTL & YLQ & A2 & Khanna R et al. ${ }^{19}$ \\
EBNA 6 & FRKAQIQGL & FRK & B37 & Rickenson and Moss \\
\hline
\end{tabular}

Abbreviations: EBNA, EBV nuclear antigen; EBV, Epstein-Barr virus; HLA, human leukocyte antigen; LMP, latent membrane protein.

The table shows the sequence, abbreviation and HLA restriction for each of the peptide epitopes used.

\section{RESULTS}

Defining the optimal LMP peptide response in NPC patients

Fifty-five patients with NPC were recruited into our study from 2003 to 2008, through the Head and Neck clinic at the Princess Alexandra Hospital, Brisbane, Queensland, Australia. Tumours were confirmed EBV-encoded RNA positive $\left(\mathrm{EBER}^{+}\right)$by in situ hybridization of paraffin sections, ${ }^{21}$ and class I human leukocyte antigen (HLA) typing was carried out. Patients with an $\mathrm{EBER}^{+}$tumour, and at least one HLA allele for which defined LMPs 1 and 2 epitopes are available (HLA A2, A11, A23/24, B40 and B57) were chosen for further analysis (26 patients). Initially, peripheral blood mononuclear cells (PBMCs) were screened for CTL specific for LMPs 1 or 2 peptides that may expand by culture for use in immunotherapy. Ex vivo PBMCs isolated from the patient's blood samples were screened for reactivity to LMPs 1 and 2 epitopes by interferon (IFN)- $\gamma$ release as detected by enzyme-linked immunospot (ELISPOT) assay (Table 2). Response to a peptide was considered positive if the number of spots elicited was more than two s.d. above the mean of three control wells of PBMC from the same patient to which no peptide or with a HLA-mismatched LMP peptide was added. Positive peptide-specific responses were found in only 6 out of the 21 patients tested, and of these four patients had positive responses to only one peptide. In addition, actual numbers of spots produced were always low.

The possibility of other LMP specificities from NPC patients that may not have been detected by ex vivo analysis was investigated by establishing bulk CTL cultures after stimulation with LMP peptides (Table 3). These cultures were established using peptide-coated LCLs rather than LCLs alone, as this generally resulted in higher levels of CTL killing when both methods were compared in eight patients (data not shown). LMP responses with $>10 \%$ specific ${ }^{51} \mathrm{Cr}$ release at an effector: target ratio of 10:1 were considered positive. LMP peptide stimulated cultures were established based on HLA-compatible LMP peptides coated on LCLs. Overall, there was little correlation between the LMP responses, as assessed by baseline ELISPOT screening of ex vivo PBMCs, compared with LMP-CTL expanded using individual synthetic LMP peptides. However, although the CTL frequency of LMP-specific $\mathrm{T}$ cells was lower than the detectable limits of the ELISPOT assay $\left(1-2 \times 10^{5}\right.$ PBMCs), by using our new protocol we were able to expand functional CTLs that were undetectable ex vivo.

Figure 1 depicts a representative result from NPC patient \#7 (HLA: A3A24B37B60), comparing ELISPOT IFN- $\gamma$ release by ex vivo PBMCs with ${ }^{51} \mathrm{Cr}$ release assays using LMP peptide-stimulated CTL as effectors. There were no significant IFN- $\gamma$ responses to the LMP 2 peptides, TYG, IED and PYL, by ex vivo PBMCs, in contrast with the EBNA 6 peptide epitope $\mathrm{FRK},{ }^{20}$ which was included as a positive control for EBV reactivity in the patient (Figure 1a). Finally, three

Table 2 Ex vivo PBMC responses from NPC patients

\begin{tabular}{lcc}
\hline Patient ID & HLA & Peptides tested \\
\hline 13 & A2 A11 B5 B51 & YLL YLQ LTA CLG FLY ALL SSC \\
10 & A2 A26 B27 B55 & YLL YLQ CLG FLY LTA \\
27 & A2 A11 B46 & YLL YLQ LTA CLG FLY ALL SSC \\
5 & A11 A24 B62 B75 & TYG SSC \\
21 & A1 A2 B46 B57 & CLG (5), FLY (8.5) \\
9 & A2 A11 B48 B75 & IAL CLG FLY ALL LLS YLL YLQ LTA \\
\hline
\end{tabular}

Abbreviations: HLA, human leukocyte antigen; PBMC, peripheral blood mononuclear cell; NPC, nasopharyngeal carcinoma.

PBMCs isolated from patient blood samples were screened for reactivity to LMP 1 and 2 epitopes by IFN- $\gamma$ release. The table shows the six NPC patients out of the 21 tested who had a measurable IFN- $\gamma$ release in an enzyme-linked immunospot (ELISPOT) assay to LMP 1 and 2 peptides.

${ }^{a} \mathrm{~A}$ positive response was greater than two s.d. above the background IFN- $\gamma$ release in response to an HLA-mismatched peptide. Numbers in brackets show spots per well for each peptide after subtracting the background (HLA-mismatched peptide). 
Table 3 LMP peptide-stimulated CTL expanded ex vivo from NPC patients

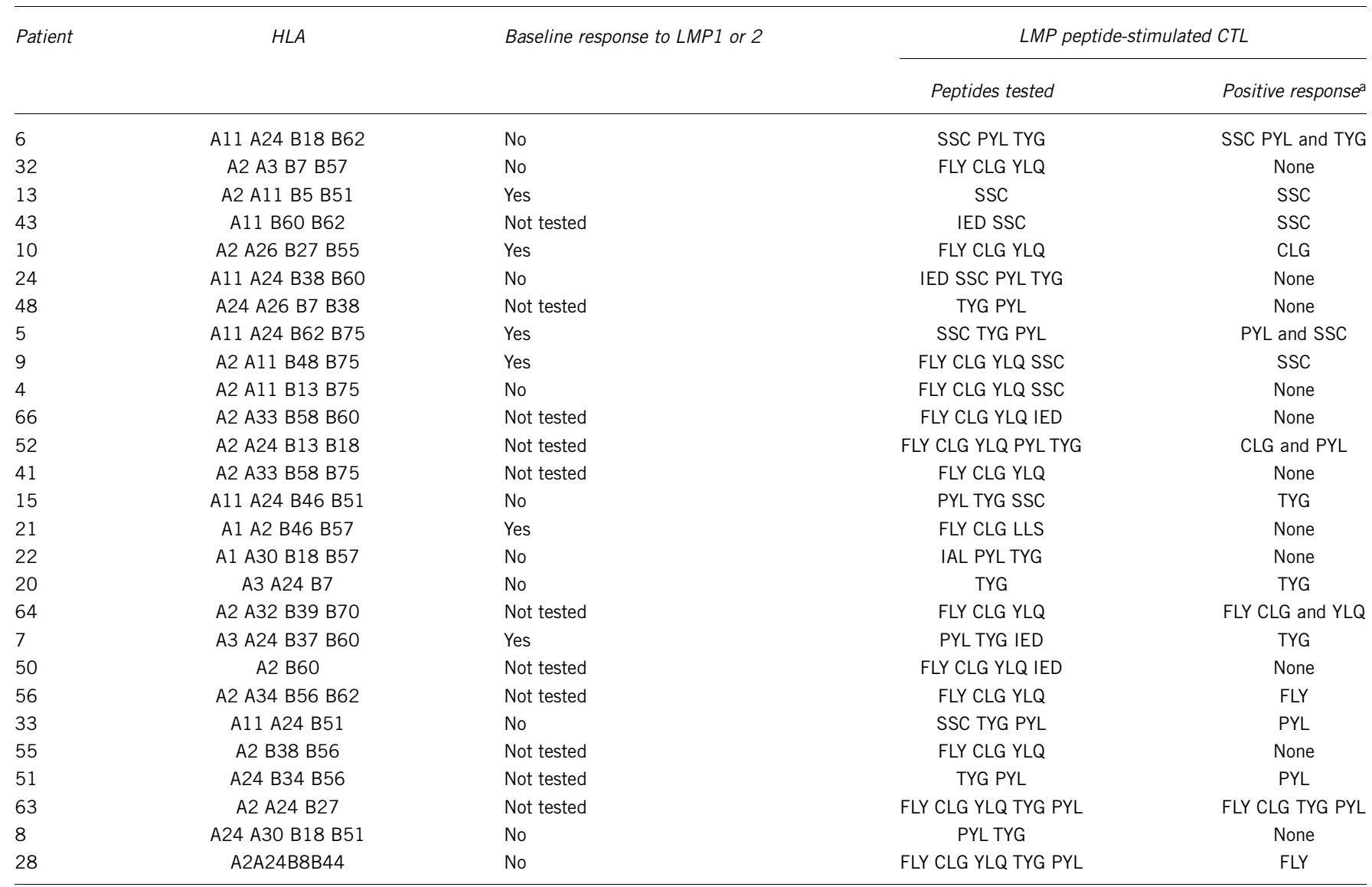

Abbreviations: CTL, cytotoxic T lymphocyte; HLA, human leukocyte antigen; LMP, latent membrane protein; NPC, nasopharyngeal carcinoma.

Cultures were established by stimulation with LMP peptides and the CTL response evaluated after 3 weeks.

The table shows that 16 NPC patients out of the 27 tested had a measurable cytotoxic response in a ${ }^{51}$ Cr release assay to LMP 1 and 2 peptides, using LMP-peptide-stimulated CTL as effector The tables
cells.

${ }^{\mathrm{a} A}$ positive response was greater than $10 \%$ specific ${ }^{51} \mathrm{Cr}$ release of autologous target cells.

separate peptide-coated LCL cultures were established using PYL, TYG and IED peptides. Only the TYG-stimulated CTL recognized peptidecoated target cells in a ${ }^{51} \mathrm{Cr}$ release assay, whereas the other cultures only recognized LCL target cells and not the specific LMP peptide they were raised against (Figure 1b). This shows that ELISPOT screening of PBMCs do not predict the optimal LMP epitope for use in expanding peptide-specific CTL in an NPC patient.

\section{Concentration of LMP peptides used for expanding LMP-specific CTL}

The concentration of peptide used to coat antigen-presenting cells for the expansion of EBV-specific CTL has not been intensively investigated, and may influence the final percentage of or avidity of CTL in culture. Routinely, peptide is added to LCLs at a final concentration of $10^{-5} \mathrm{M}\left(10 \mu \mathrm{g} \mathrm{ml}^{-1}\right)$, however, titration of a range of LMP peptides used to expand LMP-specific CTL yielded a greater number of peptide-specific $\mathrm{CD}^{+} \mathrm{T}$ cells after expansion in culture for 3 weeks. Owing to a lack of NPC patient material, some healthy donors were used to establish LMP-specific CTL cultures using variable peptide concentrations. These results were later confirmed with NPC patient blood samples (data not shown). It is worth mentioning that the level of expansion in cultures after three rounds of stimulation was sixfold for healthy individuals and also sixfold for NPC patients. It should be pointed out that there was greater variability between expansion rates in cultures established from NPC patients, ranging from 2- to 10-fold. As described in Table 4, the optimal concentration of peptides was routinely at least 100 -fold less than the standard concentration of $10^{-5} \mathrm{M}$, indicating that overstimulation of LMP-CTL cultures can lead to exhaustion of the peptide-specific CTL, perhaps through T cell$\mathrm{T}$ cell killing. ${ }^{22}$ Furthermore, the optimal concentration of LMP peptide used to expand LMP-specific CTL varied between individual peptides, ranging from $10^{-6}$ to $10^{-9} \mathrm{M}$ in this study. Figure 2 shows the improvement in expansion of the HLA A2-restricted LMP 2 peptide, FLY, in CTL grown from NPC patient \#28 (HLA: A2A24B8B44), which was dependent on the concentration of peptide used to coat the antigen-presenting cells. Both the ${ }^{51} \mathrm{Cr}$ release cytotoxicity assay (Figure 2a) and ELISPOT assay used to measure IFN- $\gamma$ release (Figure $2 \mathrm{~b}$ ) indicated that CTL stimulated with $10^{-7} \mathrm{M}$ FLY had improved avidity in the presence of reduced concentrations of FLY peptide. It should be noted that there was a concordance between the results found using the ELISPOT assay and the ${ }^{51} \mathrm{Cr}$ release cytotoxicity assay for all of the patients tested. Additionally, FLY tetramer staining confirmed that stimulation with $10^{-7} \mathrm{M}$ FLY peptide resulted in expansion of over $5 \%$ FLY-specific $\mathrm{CD}^{+} \mathrm{T}$ cells after 3 weeks in culture, compared with $10^{-5}$ and $10^{-9} \mathrm{M}$ peptide $(0.1$ and $0.2 \%$ of $\mathrm{CD}^{+}$, respectively) (Figure $2 \mathrm{c}$ ). Approximately $55 \%$ of the FLY tetramer ${ }^{+} \mathrm{CD}^{+} \mathrm{CTL}$ secreted the cytotoxic granule protease CD107 a and b (LAMP 1 and 2) in response to $10^{-7} \mathrm{M}$ FLY peptide 

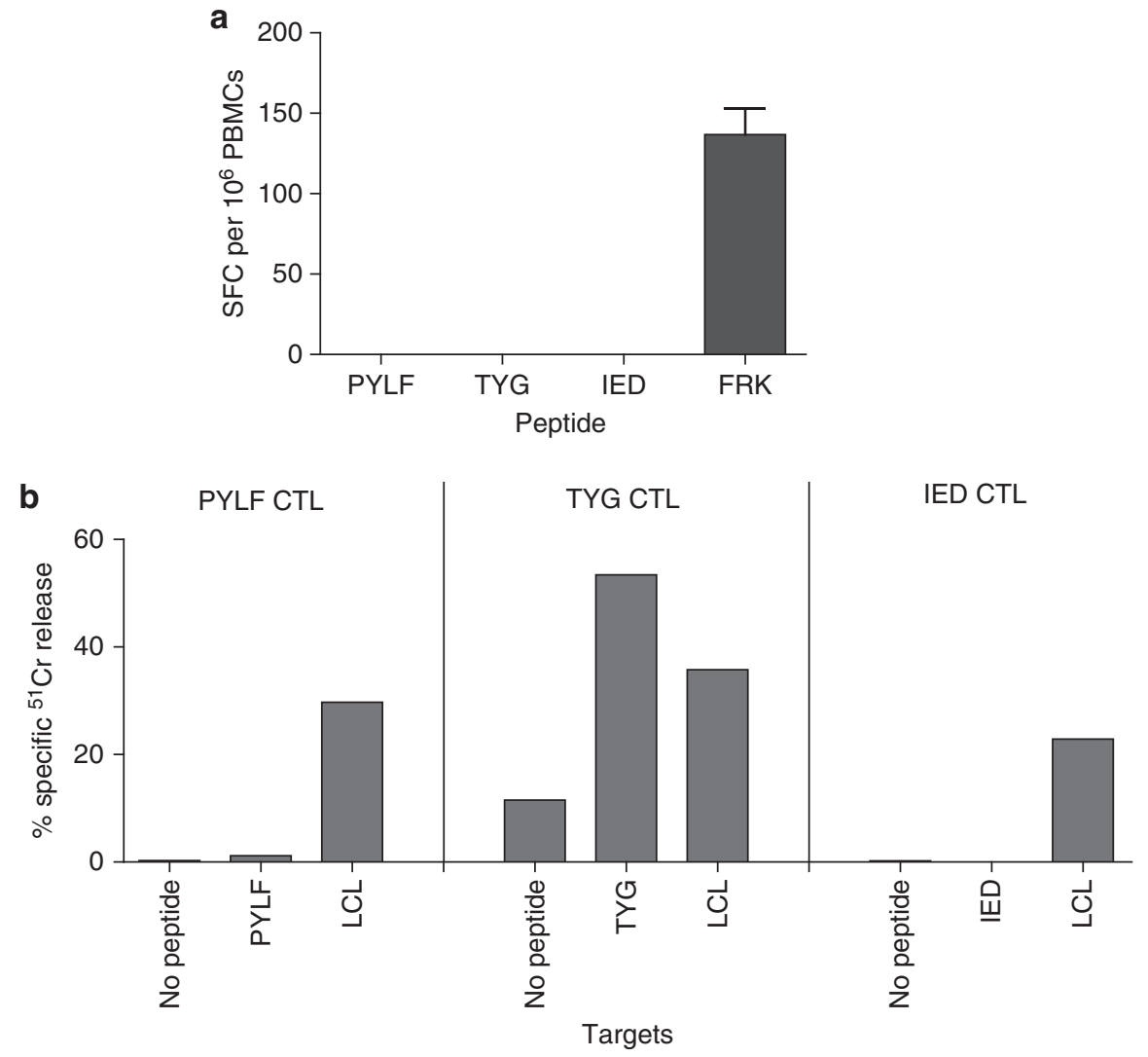

Figure 1 Comparison of ex vivo peripheral blood mononuclear cells (PBMCs) enzyme-linked immunospot (ELISPOT) with ${ }^{51} \mathrm{Cr}$ release assays derived from cytotoxic $T$ lymphocyte (CTL) expanded from a nasopharyngeal carcinoma patient. (a) PBMCs from patient \#7 (human leukocyte antigen (HLA): A3A24B37B60) were tested for interferon- $\gamma$ production in response to overnight incubation with $10^{-5} \mathrm{M}$ synthetic peptides, in an ELISPOT assay. Latent membrane protein (LMP) 2 peptides tested included HLA A24-restricted TYG, B60-restricted IED and A23-restricted PYL. Additionally, the B37-restricted Epstein-Barr virus (EBV) nuclear antigen (EBNA) 6 peptide FRK was included as a positive control for EBV latent CTL activity. Results are plotted as spot forming cells (SFCs) per $10^{6}$ PBMCs after the subtraction of control wells of PBMCs incubated without peptide, or with HLA-mismatched peptide. (b) PBMCs were stimulated weekly in vitro using LMP-peptide coated lymphoblastoid cell line (LCL) to expand LMP-specific CTL. Three different cultures were established, using PYL, TYG or IED-coated LCLs. After 3 weeks in culture the CTL were tested for LMP specificity in a ${ }^{51} \mathrm{Cr}$ release assay, using autologous PHA blasts or LCLs as target cells. The PHA blasts were coated with $10^{-5} \mathrm{M} \mathrm{LMP} 2$ peptides, and CTL were added at a 20:1 effector: target ratio, measuring ${ }^{51} \mathrm{Cr}$ release after $5 \mathrm{~h}$ incubation.

Table 4 Optimal LMP peptide concentrations for use in expanding LMP-specific CTL in vitro

\begin{tabular}{lll}
\hline Peptide & Optimal concentration $(M)$ & Tested on \\
\hline FLYALALL & $10^{-6}$ & Healthy donor \\
\hline SSCSSCPLSK & $10^{-8}$ & NPC patient \\
\hline CLGGLLTMV & $10^{-6}$ & Healthy donor \\
IEDPPFNSL & $10^{-6}$ & Healthy donor \\
\hline TYGPVFMCL & $10^{-9}$ & NPC patient \\
\hline PYLFWLAAI & $10^{-9}$ & NPC patient \\
\hline YLQQNWWTL & $10^{-6}$ & NPC patient
\end{tabular}

Abbreviations: CTL, cytotoxic T lymphocyte; LMP, latent membrane protein; NPC, nasopharyngeal carcinoma.

LMP-specific CTL cultures were established using variable peptide concentration to coat antigen-presenting cells (lymphoblastoid cell line). The table shows the optimal concentration for each peptide tested.

(Figure 2d), showing that the tetramer ${ }^{+}$cells were specifically degranulating in the presence of the peptide. The small percentage of FLY tetramer ${ }^{+} \mathrm{CD}^{+} \mathrm{CTL}$ in the $10^{-9} \mathrm{M}$ culture also degranulated at a similar rate as the cells cultured in $10^{-7} \mathrm{M}$ peptide, indicating their specificity, however there were too few tetramer ${ }^{+}$cells in the $10^{-5} \mathrm{M}$ culture to measure this (data not shown). On the basis of the variability of responses to individual LMP synthetic peptides, it is crucial to optimize the concentration of peptides used to stimulate the expansion of CTL.

\section{Determining the optimal interleukin (IL)-2 concentration in tissue culture media}

Another variable to consider in the optimization of expansion of LMP-CTL is the concentration of IL-2 in culture media. High amounts could lead to outgrowth of natural killer cells, and too little will fail to maintain $\mathrm{CD}^{+}$CTL viability and growth. Routinely, $20 \mathrm{IU}$ of rIL-2 per ml was used in our culture media, so we examined IL-2 concentrations ranging from $10 \mathrm{IU}$ to $120 \mathrm{IU} \mathrm{m}^{-1}$. Four parallel cultures were established from NPC patients with characterized LMP 2 responses to determine the optimal IL-2 concentration, using 10, 20, 60 or 120 IU IL-2 per ml. As shown in Figure 3 a using CTL expanded from NPC patient \#6 (HLA: A11A24B18B62), cell expansion numbers peaked in the cultures stimulated with $20 \mathrm{IU}$ of IL- $2 \mathrm{ml}^{-1}$, followed by 10,60 and $120 \mathrm{IU}$ of IL-2 per ml, respectively. Additionally, significant differences were observed in the phenotype of CTL, with a loss of $\mathrm{CD}^{+} \mathrm{CD}^{+} \mathrm{CTL}$ numbers in elevated IL-2 

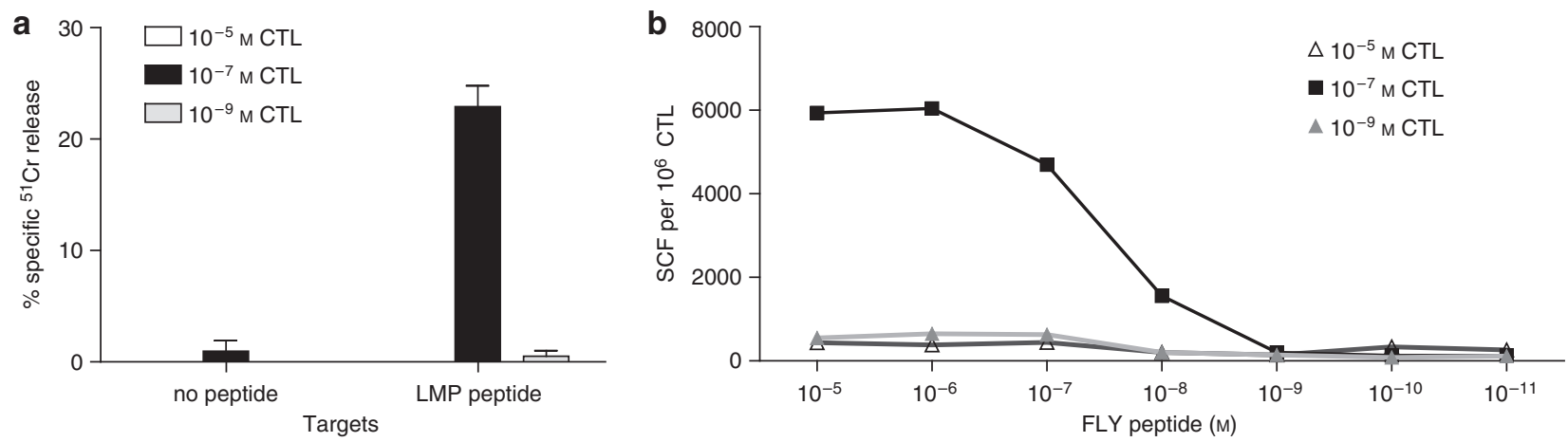

C
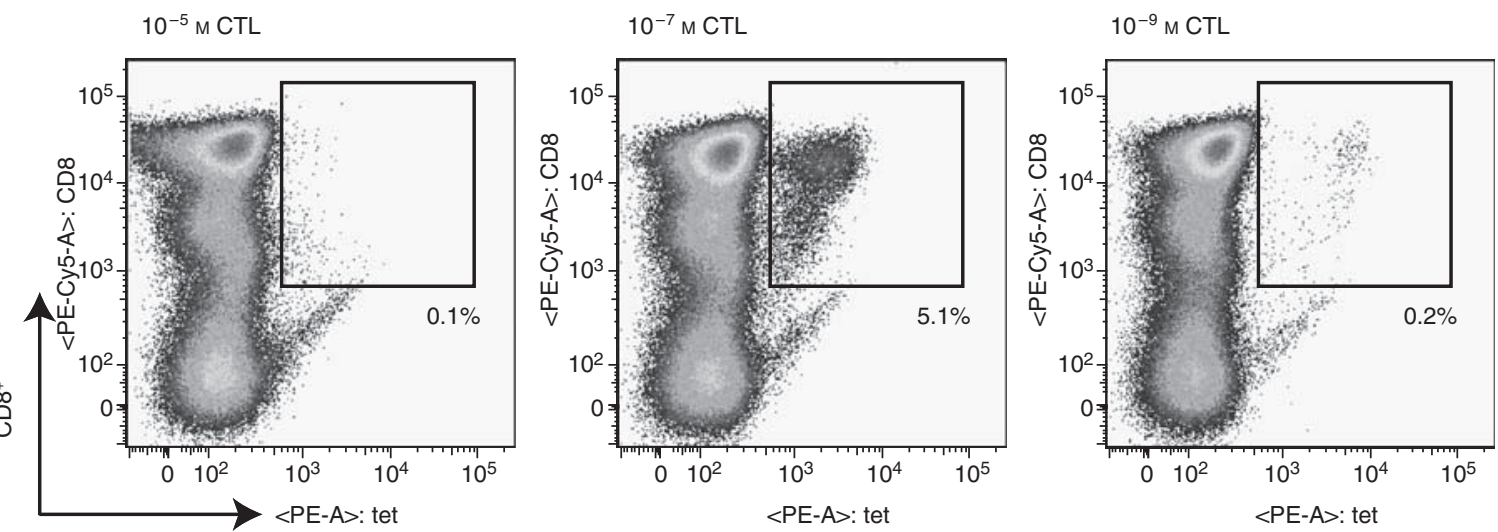

FLY tet+

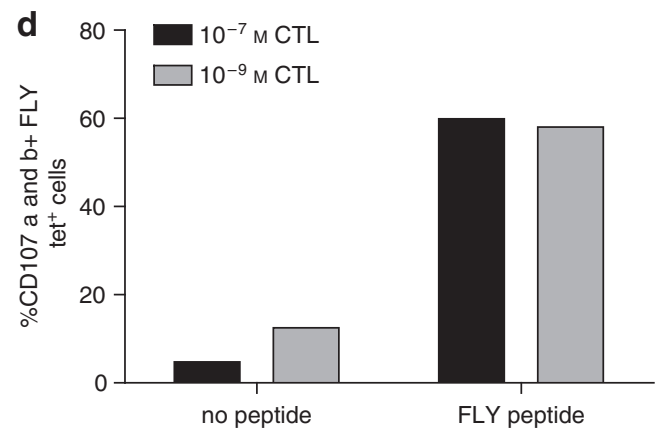

Figure 2 Optimization of the concentration of peptide used to expand FLY-specific cytotoxic T lymphocyte (CTL) from an nasopharyngeal carcinoma (NPC) patient. Peripheral blood mononuclear cells were stimulated weekly in vitro using the human leukocyte antigen (HLA) A2-restricted latent membrane protein (LMP) 2 FLY-peptide coated lymphoblastoid cell line (LCL) to expand LMP-specific CTL from NPC patient \#28 (HLA:A2A24B8B44). Individual cultures were established using different concentrations of FLY peptide to coat $L C L s$, ranging from $10^{-5}$ to $10^{-10} \mathrm{M}$, in a 10 -fold titration series. Representative data is shown for three cultures, stimulated with $10^{-5}, 10^{-7}$ or $10^{-9} \mathrm{M}$ FLY peptide, respectively. After 3 weeks in culture, the CTL were tested for FLY specificity by (a) ${ }^{51} \mathrm{Cr}$ release assay and (b) enzyme-linked immunospot (ELISPOT) assay. In the ${ }^{51} \mathrm{Cr}$ release assay, CTL were tested against autologous PHA blasts that were coated with $10^{-5} \mathrm{M}$ FLY peptide, or uncoated, at a 20:1 effector:target ratio for $5 \mathrm{~h}$. In the ELISPOT assay, CTL were incubated with decreasing concentrations of $\mathrm{FLY}$ peptide, ranging from $10^{-5}$ to $10^{-11} \mathrm{M}$, and interferon- $\gamma$ release measured after an overnight incubation. (c) The percentage of FLY+ $\mathrm{CTL}$ in the cultures was measured using FLY tetramers by flow cytometry. CTL were gated on $\mathrm{CD} 3^{+} \mathrm{CD} 8^{+}$cells, and $\mathrm{FLY}$ tetramer ${ }^{+}$cells were calculated as the percentage of $\mathrm{CD} 8^{+} \mathrm{CTL}$ (gated in the box). (d) The ability of the FLY tetramer ${ }^{+} \mathrm{CTL}$ to degranulate in response to FLY peptide was measured using CD107 a and $b$ release by flow cytometry. CTL were incubated with $2 \times 10^{-6} \mathrm{M}$ FLY peptide in the presence of CD107 a and b mAbs and Golgi Stop for $5 \mathrm{~h}$. Spontaneous release of CD107 a and b was measured in CTL incubated without peptide, compared with specific release in the presence of FLY peptide, which is plotted as a percentage of $\mathrm{CD}^{+} \mathrm{FLY}_{\text {tetramer }}^{+}$cells.

concentrations, and a subsequent increase in $\mathrm{CD}^{-} \mathrm{CD}^{-} 6^{+}$natural killer cells (Figure $3 \mathrm{~b}$ ). Further analysis showed that $26.5 \%$ of $\mathrm{CD}^{+} 8^{+}$ cells were SSC (SSCSSCPLSK)-pentamer positive in cells cultured in $10 \mathrm{IU}$ of IL-2 per $\mathrm{ml}$, in comparison with only $0.1 \%$ in $120 \mathrm{IU}$ of IL-2 per $\mathrm{ml}$ (Figure $3 \mathrm{a}$ ).

Functional assays such as ${ }^{51} \mathrm{Cr}$ and IFN- $\gamma$ release confirmed that excess amounts of IL-2 led to decreased effector cell intracellular cytokine secretion and target cell lysis in response to SSC peptide (Figures $3 \mathrm{a}$ and $\mathrm{c}$ ). Again, intracellular IFN- $\gamma$ release was highest in CTL cultured with $10 \mathrm{IU}$ of IL-2 per $\mathrm{ml}$, and was not detectable in cultures containing $120 \mathrm{IU}$ of IL-2 per ml (Figure 3a). Specific ${ }^{51} \mathrm{Cr}$ release of autologous target cells coated with SSC peptide was highest in the 10 and $20 \mathrm{IU}$ of IL-2 per ml cultured CTL compared with 60 and $120 \mathrm{IU}$ of IL-2 per ml; whereas lysis of LCL target cells was similar 

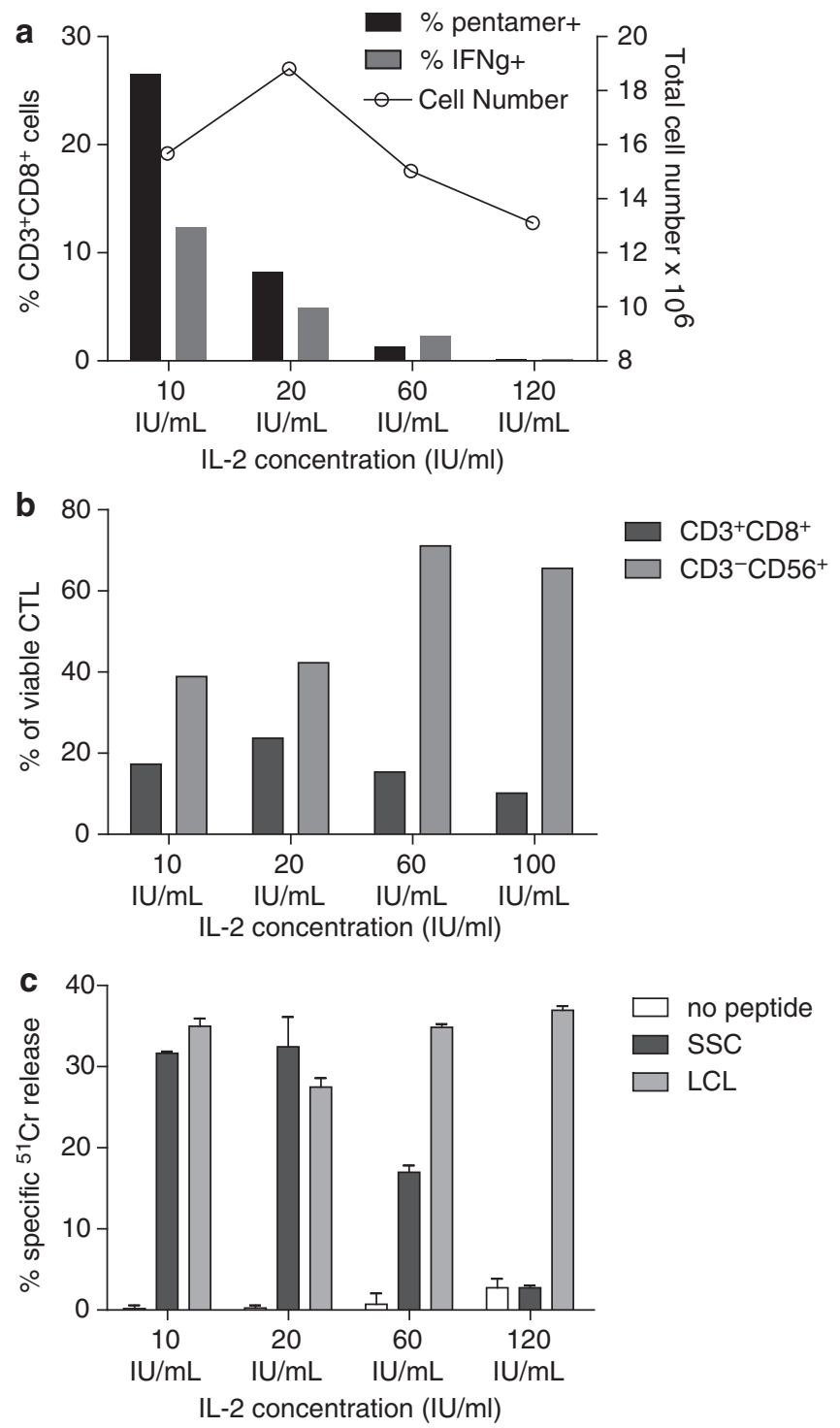

Figure 3 Determining the optimal interleukin (IL)-2 concentration in tissue culture media. Peripheral blood mononuclear cells were stimulated weekly in vitro using the human leukocyte antigen (HLA) A11-restricted latent membrane protein (LMP) 2 SSC-peptide coated lymphoblastoid cell line (LCL) to expand LMP-specific cytotoxic T lymphocyte (CTL) from nasopharyngeal carcinoma patient \#6 (HLA: A11A24B18B62). Individual cultures were established using different concentrations of IL-2 in the tissue culture media, ranging from 10 to $120 \mathrm{IU} \mathrm{ml}^{-1}$. Representative data are shown for three cultures. After 3 weeks in culture, the CTL were tested for cell expansion, phenotype, ability to secrete interferon (IFN)- $\gamma$ and ${ }^{51} \mathrm{Cr}$ release. (a) Cell cultures were counted using trypan blue exclusion to measure live cells, which were plotted against $\%$ SSC pentamer ${ }^{+}$cells, and cells that secreted IFN- $\gamma$ in response to SSC peptide (both shown as a percentage of $\mathrm{CD}^{+} 8^{+}$cells). (b) Cell phenotype was measured using CD3-FITC, CD8-PE and CD56-PeCy5 antibodies, and $\mathrm{CD}^{+} 8^{+}$ CTL compared with $\mathrm{CD}^{-} 56^{+} \mathrm{NK}$ cells. (c) In the ${ }^{51} \mathrm{Cr}$ release assay, CTL were tested against autologous PHA blasts that were coated with $10^{-5} \mathrm{~m}$ SSC peptide, or uncoated, or autologous LCLs at a 20:1 effector:target ratio for $5 \mathrm{~h}$.

between all cultures and was probably primarily natural killer cell mediated (Figure 3c). Thus, there is compelling evidence for the use of 10 or $20 \mathrm{IU}$ of IL-2 per $\mathrm{ml}$ in CTL cultures, when cell expansion, phenotype and function are examined.

\section{DISCUSSION}

Currently, the standard treatment regimen for patients with NPC is combined radiotherapy/chemotherapy, which provides favourable responses but has a poor prognosis for relapsed tumours. As NPC tumours express EBV in all undifferentiated cases, they are good candidates for CTL therapy. Indeed, recent studies have treated NPC patients with advanced disease with autologous EBV-specific CTL raised against LCLs with some success. ${ }^{7,8}$ However, there is a general consensus that targeting the LMPs 1 and 2 proteins expressed in latency II malignancies needs to be improved by activation with formulations focussed on epitopes within these proteins. This report is designed to investigate the conditions for using LMPs 1 and 2 peptide epitopes in the setting of a clinical trial.

In this study, we investigated the optimization of LMP-specific CTL expansion from NPC patients, using LMP-peptide coated LCLs as antigen-presenting cells. We have focussed this study on class I peptide epitopes as these responses are well defined and generally accepted in the field. Although there is a limited literature on class II responses, their significance is still under investigation and it would be difficult to justify their inclusion in a clinical trial. As the predominant HLA type among our NPC patients is HLA A2, we decided to screen our patients with seven HLA A2 peptides. We also attempted to cover as many non-HLA A2 responses as possible on the basis of the published data. However, it is possible that some of our patients have LMP 1- and LMP 2-specific $\mathrm{T}$ cells for peptide epitopes that have not been screened for in this study.

Several groups have reported on other strategies to generate LMPspecific $\mathrm{T}$ cells with the aim of treating patients with NPC. Firstly, autologous LCLs have been used to activate CTL responses to LMP proteins. ${ }^{6-8}$ Although some success was achieved using this protocol, our study has demonstrated that peptide-loaded LCLs are more efficient in activating a CTL response to LMP proteins (data not shown). Secondly, a vaccine consisting of dendritic cells pulsed with peptides derived from LMP 2 has been reported. ${ }^{23}$ However, the main difficulty in using this approach is that the use of dendritic cells is limited in terms of number of dendritic cells that can be isolated and the fact that their isolation is labour-intensive compared with peptidesensitized LCLs. The third approach involved the use of live viral vectors encoding either LMP proteins ${ }^{12,24}$ or a polyepitope incorporating multiple LMP-CTL epitopes. ${ }^{25,26}$ Although these latter approaches have seen some success, safety and manufacturing issues may limit their clinical application. In contrast, administration of peptides has a strong safety record and they are easy to manufacture on a large scale. Having all this in mind, we decided to test only welldefined peptides that were more likely to have a response and therefore could justify moving into clinical trials.

Not surprisingly, the expansion of immunologically weak CTL responses presented challenges earlier not observed for EBNAs 3-6 responses. Thus, we varied several aspects of LMP-specific CTL expansion, including peptide and IL-2 concentration in order to maximize CTL yield within 3 weeks of culture. Initially we hypothesized that selection of the optimal LMP peptide for use in establishing an LMP-specific CTL culture from NPC patients, when PBMC numbers are often limited, may be achieved using a screening assay such as ELISPOT assay. However, we found little correlation between IFN- $\gamma$ production by ex vivo PBMCs in response to LMP peptides, and cytotoxic assays using CTL expanded with peptidecoated LCLs. In particular, the NPC patient described in Figure 1 had no measurable LMP-specific CTL in ex vivo PBMCs as tested by ELISPOT assay. However, when LMP peptides were used to expand CTL, the patient responded to the A24-restricted LMP 2 peptide TYG 
and had no response to either the IED or PYLF LMP 2 peptides. Therefore, the selection of the optimal LMP peptide for expansion of LMP-specific CTL should be on the basis of a series of small-scale cultures using the available LMP peptides based on a patient's HLA type, before increasing the culture size for the expansion of CTL for use in adoptive immunotherapy.

The concentration of peptide used to stimulate an LMP-specific CTL culture has not been widely investigated, and routinely $10^{-5} \mathrm{M}$ $\left(10 \mu \mathrm{g} \mathrm{ml}^{-1}\right)$ is used to coat LCLs used as antigen-presenting cells. However, we considered the possibility that excess peptide added to immunologically weak responses may decrease the number of LMPCTL in culture over time. The phenomenon of $\mathrm{T}$ cell- $\mathrm{T}$ cell killing showed that excessive amounts of peptide bound to one CTL may be recognized and killed by another peptide-specific CTL. In order to address this, LMP-specific CTL cultures were established using 10-fold titrations of peptide used to coat LCLs, ranging from $10^{-5}$ to $10^{-10} \mathrm{M}$. Interestingly, we found that each LMP peptide tested had a variable optimal concentration for the expansion of LMPspecific CTL over a period of 3 weeks. A single log difference in concentration of LMP peptide used to stimulate the culture had profound effects on the final percentage of peptide-specific $\mathrm{CD}^{+}$ CTL, with up to a 20-fold increase observed for some peptides. Generally, CTL expanded with reduced peptide concentrations also had improved affinity for the peptide, as observed in ELISPOT assays in which CTL were tested against decreasing concentrations of peptide. Using optimized peptide concentrations, we had successful CTL expansion from 16 out of the 27 NPC patients tested. Thus, it is imperative that peptide concentrations should be optimized, especially for the expansion of immunologically weak CTL responses to maximize peptide-specific CTL yield.

Considering the variability in percentage of LMP-peptide-specific CTL obtained by reducing peptide on antigen-presenting cells, we also optimized the IL-2 concentration used in tissue culture media. Routinely, our laboratory used 20 IU of IL- 2 per ml media during the 3 week culture period, and we found that this concentration was optimal when cell expansion, percentage of peptide-specific cells and capacity to kill peptide-coated target cells was measured. Certainly, increasing the concentration of IL-2 beyond $20 \mathrm{IU} \mathrm{ml}^{-1}$ was counterproductive, particularly because of an expansion of $\mathrm{CD}^{2} 6^{+}$natural killer cells in the culture. It is worth mentioning that somewhat unexpectedly and unlike healthy donors, we needed to add IL-2 at the time of setting up the cultures because, otherwise, it was extremely difficult to generate a CTL response. The reason for this early requirement for IL-2 is unclear.

Overall, our studies show that the culture conditions for expansion of CTL need to be tailored for each patient, as there is not a universal protocol that can be used for every donor. Specifically, each peptide showed an optimal concentration for the expansion of LMP-specific CTL.

As highlighted above, there is an increasing demand for improved strategies to target latency II tumours, such as NPC and HL. Having established the best culture conditions for activation of CTLs, it is our intention to conduct a clinical trial that has been optimized to allow the activation of a CTL response for adoptive transfer of CTL in NPC patients.

\section{METHODS}

\section{Patient selection}

Healthy laboratory and newly diagnosed or relapsed NPC patients were enroled into the study, through the Head and Neck Clinic at the Princess Alexandra Hospital in Brisbane, Queensland, Australia. Each blood donor was assigned a unique identification code and provided informed consent. This study was approved by the institutional Human Research Ethics Committee, which conforms to the Declaration of Helsinki.

\section{Enzyme-linked immunospot assays}

The enzyme-linked immunospot assays used to measure the frequencies of EBV epitope-specific CTL in PBMCs or cultured cells were done as described earlier. ${ }^{27} 2 \times 10^{5}$ PBMCs or $5 \times 10^{4}$ cultured CTL were added per well in triplicate and stimulated with synthetic peptides $\left(10^{-5}-10^{-10} \mathrm{M}\right)$. Peptidespecific spots were calculated after subtraction of the control wells consisting of PBMCs without added peptide or HLA-mismatched peptide. Generally, the number of spots in these control wells was $<5$.

\section{Establishing LMP-specific CTL cultures}

Cytotoxic T-lymphocyte cultures were established using peptide-coated the autologous LCLs. Cultures were maintained in RPMI with $10 \%$ fetal bovine serum and various amounts of IL-2 (as stated for each experiment) at $37^{\circ} \mathrm{C}$ with $5 \% \mathrm{CO}_{2}$ and $95 \%$ relative humidity. In either case, PBMCs were isolated from blood samples from healthy individuals or from NPC patients collected in EDTA. LMP peptide expanded CTL were established with $\gamma$-irradiated LCLs coated with synthetic peptides at a range of concentrations for $1 \mathrm{~h}$ at $37^{\circ} \mathrm{C}$, washed three times and then added to patient-matched PBMCs at a 30:1 ratio. IL-2 was added to these cultures at the times and concentrations stated for each experiment. CTL were restimulated weekly as described for day 1 and tested on day 21 .

\section{${ }^{51} \mathrm{Cr}$ release assays}

The ability of LMP-specific CTL to induce lysis was measured by specific ${ }^{51} \mathrm{Cr}$ release of LMP peptide-coated target cells. The targets used were phytohaemaglutinin (PHA)-stimulated PBMCs (PHA-blasts). Autologous PHA-blasts were incubated with synthetic peptides for $1 \mathrm{~h}$ at $37^{\circ} \mathrm{C}$ with $5 \% \mathrm{CO}_{2}$, washed once, then incubated with ${ }^{51} \mathrm{Cr}$ for $1 \mathrm{~h}$ at $37^{\circ} \mathrm{C}$ with $5 \% \mathrm{CO}_{2}$, washed twice, and resuspended at $10^{5}$ cells per $\mathrm{ml}$ in RPMI, $10 \%$ fetal bovine serum. $10^{4}$ of these target cells were added to a well of a 96-well plate, and effector CTL were added at a range of effector:target ratios. The plates were incubated at $37^{\circ} \mathrm{C}$ with $5 \%$ $\mathrm{CO}_{2}$ for $5 \mathrm{~h}$, then centrifuged to pellet the cells and $25 \mu \mathrm{l}$ of supernatant was collected into Luma-Plate 96-well plates (Packard, Packard Instrument Co, Connecticut, CT, USA). The plates were dried and the amounts of ${ }^{51} \mathrm{Cr}$ were determined for each supernatant, using a TopCount microplate scintillation counter (Packard). The percentage of specific lysis was calculated by the formula:

$$
\begin{aligned}
& {[(\text { mean specific release } \pm \text { mean spontaneous release }) /} \\
& \text { mean total release }] \times 100
\end{aligned}
$$

Phenotypic analysis, tetramer and intracellular cytokine staining The phenotype of both PBMCs and LMP-specific CTL was determined by labelling cells with fluorochrome-labelled monoclonal antibodies specific for T-cell markers. Cells $\left(2 \times 10^{5}\right)$ were washed once in flow cytometry (FACS) buffer ( $1 \times$ Phosphate-buffered saline, $2 \%$ fetal bovine serum) and resuspended in $50 \mu \mathrm{l}$ FACs buffer. CD3-fluorescein isothiocyanate (FITC), CD4-PeCy5 (Beckman Coulter, Fullerton, CA, USA), CD8-phycoerythrin (PE), CD56PeCy5, CD25-PE (BD Pharmingen, San Diego, CA, USA, Becton Dickinson, Franklin Lakes, NJ, USA) were added to the cells at a $1 / 20$ dilution and incubated on ice for $30 \mathrm{~min}$, washed once, resuspended in FACs buffer and analysed on a FACSCanto (Becton Dickinson). CTL were assessed for their ability to secrete IFN- $\gamma$ by intracellular cytokine staining and/or mobilize CD107 $\mathrm{a}$ and $\mathrm{b}$ to the surface ${ }^{28}$ in response to incubation with LMP peptides. ${ }^{29}$ $5 \times 10^{5}$ CTL were resuspended in $200 \mu$ l of FACS buffer, $2 \mu$ of Golgi stop (BD Biosciences, San Jose, CA, USA) diluted 1/30, $1 \mu \mathrm{l}$ each of anti-CD107aFITC and anti-CD107b-FITC, and LMP peptide $\left(10^{-5}-10^{-9} \mathrm{M}\right.$ final concentration). Cells were incubated at $37^{\circ} \mathrm{C}$ and $5 \% \mathrm{CO}_{2}$ for $5 \mathrm{~h}$, then centrifuged and washed twice in $200 \mu \mathrm{l}$ of FACS buffer. PE-labelled peptide-major histocompatibility complex tetramer staining was performed at a 1/100 dilution in FACS buffer for $30 \mathrm{~min}$ at room temperature, and then cells were washed twice. Cells were fixed in $40 \mu \mathrm{l}$ of $1 \%$ paraformaldehyde on ice for $20 \mathrm{~min}$, washed twice, and incubated overnight at $4{ }^{\circ} \mathrm{C}$ in $40 \mu \mathrm{l}$ of $0.25 \%$ saponin, and monoclonal antibodies to detect CD3, CD8 and IFN- $\gamma$. Cells were washed twice before 
acquisition of data with a FACSCanto (BD Biosciences). Data analysis was done using FlowJo (Treestar, Ashland, OR, USA).

\section{ACKNOWLEDGEMENTS}

Supported by the Garnett Passe and Rodney Williams Memorial Foundation project grant, the Queensland Cancer Fund project grant, the NH\&MRC Peter Doherty postdoctoral training fellowship (J Davis).

1 Rickinson AB, Kieff E. In: Fields BN, Knipe DM, Howley PM (eds). Epstein-Barr Virus: Fields Virology. Lippincott-Raven Publishers: Philadelphia, 1996, pp 2397-2446.

2 Haque T, Taylor C, Wilkie GM, Murad P, Amlot PL, Beath S et al. Complete regression of posttransplant lymphoproliferative disease using partially HLA-matched Epstein Barr virus-specific cytotoxic T cells. Transplantation 2001; 72: 1399-1402.

3 Rooney CM, Smith CA, Ng CYC, Loftin S, Li C, Krance RA et al. Use of gene-modified virus-specific $T$ lymphocytes to control Epstein-Barr-virus-related lymphoproliferation. Lancet 1995; 345: 9-13.

4 Sherritt MA, Bharadwaj M, Burrows JM, Morrison LE, Elliott SL, Davis JE et al. Reconstitution of the latent T-lymphocyte response to Epstein-Barr virus is coincident with long-term recovery from posttransplant lymphoma after adoptive immunotherapy. Transplantation 2003; 75: 1556-1560.

5 Straathof KC, Leen AM, Buza EL, Taylor G, Huls MH, Heslop HE et al. Characterization of latent membrane protein 2 specificity in CTL lines from patients with EBV-positive nasopharyngeal carcinoma and lymphoma. J Immunol 2005; 175: 4137-4147.

6 Chua D, Huang J, Zheng B, Lau SY, Luk W, Kwong DL et al. Adoptive transfer of autologous Epstein-Barr virus-specific cytotoxic $T$ cells for nasopharyngeal carcinoma. Int J Cancer 2001; 94: 73-80.

7 Comoli P, Pedrazzoli P, Maccario R, Basso S, Carminati O, Labirio M et al. Cell therapy of stage IV nasopharyngeal carcinoma with autologous Epstein-Barr virus-targeted cytotoxic T lymphocytes. J Clin Oncol 2005; 23: 8942-8949.

8 Straathof KC, Bollard CM, Popat U, Huls MH, Lopez T, Morriss MC et al. Treatment of nasopharyngeal carcinoma with Epstein-Barr virus-specific T lymphocytes. Blood 2005; 105: 1898-1904.

9 Su Z, Peluso MV, Raffegerst SH, Schendel DJ, Roskrow MA. The generation of LMP2aspecific cytotoxic $T$ lymphocytes for the treatment of patients with Epstein-Barr viruspositive Hodgkin disease. Eur J Immunol 2001; 31: 947-958.

10 Gahn B, Siller-Lopez F, Pirooz AD, Yvon E, Gottschalk S, Longnecker R et al. Adenoviral gene transfer into dendritic cells efficiently amplifies the immune response to LMP2A antigen: a potential treatment strategy for Epstein-Barr virus-positive Hodgkin's lymphoma. Int J Cancer 2001; 93: 706-713.

11 Gottschalk S, Edwards OL, Sili U, Huls MH, Goltsova T, Davis AR et al. Generating CTLs against the subdominant Epstein-Barr virus LMP1 antigen for the adoptive immunotherapy of EBV-associated malignancies. Blood 2003; 101: 1905-1912.

12 Bollard CM, Straathof KC, Huls MH, Leen A, Lacuesta K, Davis A et al. The generation and characterization of LMP2-specific CTLs for use as adoptive transfer from patients with relapsed EBV-positive Hodgkin disease. J Immunother 2004; 27: 317-327.

13 Duraiswamy J, Burrows JM, Bharadwaj M, Burrows SR, Cooper L, Pimtanothai N et al. Ex vivo analysis of T-cell responses to Epstein-Barr virus-encoded oncogene latent membrane protein 1 reveals highly conserved epitope sequences in virus isolates from diverse geographic regions. J Virol 2003; 77: 7401-7410.
14 Lee SP, Thomas WA, Murray RJ, Khanim F, Kaur S, Young LS et al. HLA A2.1restricted cytotoxic $T$ cells recognizing a range of Epstein-Barr virus isolates through a defined epitope in latent membrane protein LMP2. J Virol 1993; 67: 7428-7435.

15 Lautscham G, Haigh T, Mayrhofer S, Taylor G, Croom-Carter D, Leese A et al. Identification of a TAP-independent, immunoproteasome-dependent CD8+ T-cell epitope in Epstein-Barr virus latent membrane protein 2. J Virol 2003; 77: 2757-2761.

16 Lee SP, Tierney RJ, Thomas WA, Brooks JM, Rickinson AB. Conserved CTL epitopes within EBV latent membrane protein 2: a potential target for CTL-based tumor therapy. $\mathrm{J}$ Immunol 1997; 158: 3325-3334.

17 Lee SP, Chan AT, Cheung ST, Thomas WA, CroomCarter D, Dawson CW et al. CTL control of EBV in nasopharyngeal carcinoma (NPC): EBV-specific CTL responses in the blood and tumors of NPC patients and the antigen-processing function of the tumor cells. J Immunol 2000; 165: 573-582.

18 Khanna R, Burrows SR, Moss DJ, Silins SL. Peptide transporter (TAP-1 and TAP-2)independent endogenous processing of Epstein-Barr virus (EBV) latent membrane protein 2A: implications for cytotoxic T-lymphocyte control of EBV-associated malignancies. J Virol 1996; 70: 5357-5362.

19 Khanna R, Burrows SR, Nicholls J, Poulsen LM. Identification of cytotoxic T cell epitopes within Epstein-Barr virus (EBV) oncogene latent membrane protein 1 (LMP1): evidence for HLA A2 supertype-restricted immune recognition of EBV-infected cells by LMP1-specific cytotoxic T lymphocytes. Eur J Immunol 1998; 28: 451-458.

20 Rickinson AB, Moss DJ. Human cytotoxic T lymphocyte responses to Epstein-Barr virus infection. Annu Rev Immunol 1997; 15: 405-431.

21 Niedobitek G, Young LS, Sam CK, Brooks L, Prasad U, Rickinson AB. Expression of Epstein-Barr virus genes and of lymphocyte activation molecules in undifferentiated nasopharyngeal carcinomas. Am J Pathol 1992; 140: 879-887.

22 Burrows SR, Fernan A, Argaet V, Suhrbier A. Bystander apoptosis induced by CD8+ cytotoxic T cell (CTL) clones: implications for CTL Iytic mechanisms. Int Immunol 1993; 5: 1049-1058.

23 Lin CL, Lo WF, Lee TH, Ren Y, Hwang SL, Cheng YF et al. Immunization with Epstein-Barr Virus (EBV) peptide-pulsed dendritic cells induces functional CD8+ T-cell immunity and may lead to tumor regression in patients with EBV-positive nasopharyngeal carcinoma. Cancer Res 2002; 62: 6952-6958.

24 Taylor GS, Haigh TA, Gudgeon NH, Phelps RJ, Lee SP, Steven NM et al. Dual stimulation of Epstein-Barr Virus (EBV)-specific CD4+- and CD8+-T-cell responses by a chimeric antigen construct: potential therapeutic vaccine for EBV-positive nasopharyngeal carcinoma. J Virol 2004; 78: 768-778.

25 Duraiswamy J, Sherritt M, Thomson S, Tellam J, Cooper L, Connolly G et al. Therapeutic LMP1 polyepitope vaccine for EBV-associated Hodgkin disease and nasopharyngeal carcinoma. Blood 2003; 101: 3150-3156.

26 Duraiswamy J, Bharadwaj M, Tellam J, Connolly G, Cooper L, Moss D et al. Induction of therapeutic T-cell responses to subdominant tumor-associated viral oncogene after immunization with replication-incompetent polyepitope adenovirus vaccine. Cancer Res 2004; 64: 1483-1489.

27 Bharadwaj M, Parsons PG, Moss DJ. Cost-efficient quantification of enzyme-linked immunospot. Biotechniques 2001; 30: 36-38.

28 Betts MR, Brenchley JM, Price DA, De Rosa SC, Douek DC, Roederer M et al. Sensitive and viable identification of antigen-specific CD8+ T cells by a flow cytometric assay for degranulation. J Immunol Methods 2003; 281: 65-78.

29 Moor RJ, Morrison LE, Moss DJ, Tscharke DC. Use of CD107-based cell sorting ex vivo to enrich subdominant CD8+ T cells in culture. Immunol Cell Biol 2007; 85: $546-550$ 